\title{
Bipolar fuzzy ideals of Near Rings
}

\author{
Hyoung Gu Baik \\ School of Computer and Information, Ulsan Colleage
}

\begin{abstract}
Based on the theory of a bipolar fuzzy set, the notion of a bipolar fuzzy subring/ideal of a Near ring is introduced and related properties are investigated. Characterizations of a bipolar fuzzy subnear ring and a bipolar fuzzy ideal in near ring are established. Relations between a bipolar fuzzy ideal and a level cut are discussed. Using bipolar fuzzy ideals, we discuss characterizations of Noetherian Near ring.
\end{abstract}

Key Words: Near Ring, Biploar fuzzy set, Biploar fuzzy ideal

\section{Introduction}

Zadeh [14] introduced the concept of fuzzy set as a new mathematical tool for dealing with uncertainties. There are several kinds of fuzzy set extensions in the fuzzy set theory, for example, intuitionistic fuzzy sets, interval-valued fuzzy sets, rough fuzzy set, soft fuzzy set, vague sets, etc $[5,11,12]$. Bipolar-valued fuzzy set is another extension of fuzzy set whose membership degree range is extended from the interval $[0,1]$ to the interval $[-1,1]$. The idea of bipolar valued fuzzy set was introduced by K.M.Lee [9, 10], as a generalization of the notion of fuzzy set. Since then, the theory of bipolar valued fuzzy sets has become a vigorous area of research in different disciplines such as algebraic structure, medical science, graph theory, decision making, machine theory and so on [3, 4, 6, 7, 8]. S. Abou-Zaid [2] introduced the notion of a fuzzy subnear-ring, and studied fuzzy left (resp. right) ideals of a near-ring, and gave some properties of fuzzy prime ideals of a near-ring. In this paper, we introduce the notion of bipolar fuzzy subring/ideal of a Near ring and investigate its properties.

\section{Preliminaries}

By a near-ring [13] we mean a non-empty set $R$ with two binary operations + and - satisfying the following axioms:

(1) $(R,+)$ is a group(not necessarily abelian),

(2) $(R, \cdot)$ is a semigroup,

(3) $x \cdot(y+z)=x \cdot y+x \cdot z$ for all $x, y, z \in R$.
Precisely speaking, it is a left near-ring because it satisfies the left distributive law. We will use the word near-ring in stead of left near-ring. We denote $x y$ instead of $x \cdot y$. Note that $x 0=0$ and $x(-y)=-x y$ but in general $0 x \neq 0$ for some $x \in R$.

An ideal $I$ of a near-ring $R$ is a subset of $\mathrm{R}$ such that

(1) $(I,+)$ is a normal subgroup of $(R ;+)$,

(2) $R I \subset I$,

(3) $(r+i) s-r s \in I$ for any $i \in I$ and any $r, s \in R$.

Note that $I$ is a left ideal of $R$ if $I$ satisfies (1) and (2), and $I$ is a right ideal of $R$ if $I$ satisfies (1) and (3).

Let $X$ be the universe of discourse. A bipolar-valued fuzzy set $\Phi$ in $X$ is an object having the form

$$
\Phi=\left\{\left(x, \mu_{\Phi}^{+}(x), \mu_{\Phi}^{-}(x)\right) \mid x \in X\right\}
$$

where $\mu_{\Phi}^{+}: X \rightarrow[0,1]$ and $\mu_{\Phi}^{-}: X \rightarrow[-1,0]$ are mappings. The positive membership degree $\mu_{\Phi}^{+}(x)$ denoted the satisfaction degree of an element $x$ to the property corresponding to a bipolar-valued fuzzy set $\Phi=\left\{\left(x, \mu_{\Phi}^{+}(x), \mu_{\Phi}^{-}(x)\right) \mid x \in X\right\}$, and the negative membership degree $\mu_{\Phi}^{-}(x)$ denotes the satisfaction degree of $x$ to some implicit counter-property of $\Phi=$ $\left\{\left(x, \mu_{\Phi}^{+}(x), \mu_{\Phi}^{-}(x)\right) \mid x \in X\right\}$.

For a bipolar fuzzy set $\Phi=\left(X ; \mu_{\Phi}^{+}, \mu_{\Phi}^{-}\right)$and $(t, s) \in$ $[-1,0] \times[0,1]$, we define

$$
\begin{aligned}
& \Phi_{t}^{P}:=\left\{x \in X \mid \mu_{\Phi}^{+}(x) \geq t\right\} \\
& \Phi_{s}^{N}:=\left\{x \in X \mid \mu_{\Phi}^{-}(x) \leq s\right\}
\end{aligned}
$$


which are called the positive t-cut of $\Phi=\left(X ; \mu_{\Phi}^{+}, \mu_{\Phi}^{-}\right)$and the negative $s$-cut of $\Phi=\left(X ; \mu_{\Phi}^{+}, \mu_{\Phi}^{-}\right)$, respectively. For $(s, t) \in[-1,0] \times[0,1]$, the set

$$
\Phi_{(t, s)}:=\Phi_{t}^{P} \cap \Phi_{s}^{N}
$$

is called the $(t, s)$-cut of $\Phi=\left(X ; \mu_{\Phi}^{+}, \mu_{\Phi}^{-}\right)$.

\section{Bipolar fuzzy subring and bipolar fuzzy ideals}

In what follows, let $R$ denote a Near-ring unless otherwise specified.

Definition 3.1. A bipolar fuzzy set $\Phi=\left(R ; \mu_{\Phi}^{+}, \mu_{\Phi}^{-}\right)$in $R$ is called a bipolar fuzzy subnear ring of $R$ if for all $x, y \in R$,

(BF1) $\mu_{\Phi}^{+}(x-y) \geq \min \left\{\mu_{\Phi}^{+}(x), \mu_{\Phi}^{+}(y)\right\}, \mu_{\Phi}^{-}(x-y) \leq$ $\max \left\{\mu_{\Phi}^{-}(x), \mu_{\Phi}^{-}(y)\right\}$

(BF2) $\mu_{\Phi}^{+}(x y) \geq \min \left\{\mu_{\Phi}^{+}(x), \mu_{\Phi}^{+}(y)\right\}, \mu_{\Phi}^{-}(x y) \leq$ $\max \left\{\mu_{\Phi}^{-}(x), \mu_{\Phi}^{-}(y)\right\}$

Definition 3.2. A bipolar fuzzy subnear ring $\Phi=$ $\left(R ; \mu_{\Phi}^{+}, \mu_{\Phi}^{-}\right)$in $R$ is called a bipolar fuzzy ideal of $R$ if for all for all $x, y, z \in R$,

(BF3) $\mu_{\Phi}^{+}(y+x-y) \geq \mu_{\Phi}^{+}(x), \mu_{\Phi}^{-}(y+x-y) \leq \mu_{\Phi}^{-}(x)$,

(BF4) $\mu_{\Phi}^{+}(x y) \geq \mu_{\Phi}^{+}(y), \mu_{\Phi}^{-}(x y) \leq \mu_{\Phi}^{-}(y)$

(BF5) $\mu_{\Phi}^{+}((x+z) y-x y) \geq \mu_{\Phi}^{+}(z), \mu_{\Phi}^{-}((x+z) y-x y) \leq$ $\mu_{\Phi}^{-}(z)$

Note that $A$ is a left biploar fuzzy ideal of $R$ if $A$ satisfies (BF1),(BF2),(BF3) and (BF4), and $A$ is a right biploar fuzzy ideal of $R$ if $I$ satisfies (BF1),(BF2),(BF3) and (BF5).

Proposition 3.3. If $\Phi=\left(R ; \mu_{\Phi}^{+}, \mu_{\Phi}^{-}\right)$is a bipolar fuzzy subnear ring of $R$, then $\mu_{\Phi}^{+}(0) \geq \mu_{\Phi}^{+}(x)$ and $\mu_{\Phi}^{-}(0) \leq$ $\mu_{\Phi}^{-}(x)$ for all $x \in R$.

Proof. Let $x \in R$. Then

$$
\mu_{\Phi}^{+}(0)=\mu_{\Phi}^{+}(x-x) \geq \min \left\{\mu_{\Phi}^{+}(x), \mu_{\Phi}^{+}(x)\right\}=\mu_{\Phi}^{+}(x)
$$

and

$$
\mu_{\Phi}^{-}(0)=\mu_{\Phi}^{-}(x-x) \leq \max \left\{\mu_{\Phi}^{-}(x), \mu_{\Phi}^{-}(x)\right\}=\mu_{\Phi}^{-}(x) .
$$

This completes the proof.

In the following Theorem, we give an example of bipolar fuzzy ideal of $R$
Theorem 3.4. Let $I$ be an ideal of near ring $R$. Suppose the $\Phi=\left(X ; \mu_{\Phi}^{+}, \mu_{\Phi}^{-}\right)$is a bipolar fuzzy subset defined by $\Phi=\left(X ; \mu_{\Phi}^{+}, \mu_{\Phi}^{-}\right)$in $R$ by

$$
\mu_{\Phi}^{+}(x):= \begin{cases}p_{1} & \text { if } x \in I \\ p_{2} & \text { if } x \notin I\end{cases}
$$

and

$$
\mu_{\Phi}^{-}(x):= \begin{cases}n_{1} & \text { if } x \in I, \\ n_{2} & \text { if } x \notin I,\end{cases}
$$

for $p_{1}, p_{2} \in[0,1]$ and $n_{1}, n_{2} \in[-1,0]$ such that $p_{1}>p_{2}$ and $n_{1}<n_{2}$. Then $\Phi=\left(R ; \mu_{\Phi}^{+}, \mu_{\Phi}^{-}\right)$is an bipolar fuzzy ideal of $R$.

Proof. Let $x, y \in R$. If $x, y \in I$, then $x-y \in I$. It follows that $\mu_{\Phi}^{+}(x-y)=p_{1}=\min \left\{\mu_{\Phi}^{+}(x), \mu_{\Phi}^{+}(y)\right\}$ and $\mu_{\Phi}^{-}(x-y)=n_{1}=\max \left\{\mu_{\Phi}^{-}(x), \mu_{\Phi}^{-}(y)\right\}$, Also we have $\mu_{\Phi}^{+}(x y)=p_{1}=\min \left\{\mu_{\Phi}^{+}(x), \mu_{\Phi}^{+}(y)\right\}$ and $\mu_{\Phi}^{-}(x y)=n_{1}=$ $\max \left\{\mu_{\Phi}^{-}(x), \mu_{\Phi}^{-}(y)\right\}$,

If $x$ or $y$ is not contained in $I$, then $\mu_{\Phi}^{+}(x-$ $y) \geq p_{2}=\min \left\{\mu_{\Phi}^{+}(x), \mu_{\Phi}^{+}(y)\right\}, \quad \mu_{\Phi}^{-}(x-$ $y) \leq n_{2}=\max \left\{\mu_{\Phi}^{-}(x), \mu_{\Phi}^{-}(y)\right\}, \mu_{\Phi}^{+}(x y) \geq$ $p_{2}=\min \left\{\mu_{\Phi}^{+}(x), \mu_{\Phi}^{+}(y)\right\}$ and $\mu_{\Phi}^{-}(x y) \leq n_{2}=$ $\max \left\{\mu_{\Phi}^{-}(x), \mu_{\Phi}^{-}(y)\right\}$, Therefore $\Phi=\left(X ; \mu_{\Phi}^{+}, \mu_{\Phi}^{-}\right)$satisfies (BF1) and (BF2).

Let $x, y \in R$. We discuss the following two cases: If $x \in I$ Then $y+x-y \in I$. Thus $\mu_{\Phi}^{+}(y+x-y)=$ $p_{1}=\mu_{\Phi}^{+}(x), \mu_{\Phi}^{-}(y+x-y)=n_{1}=\mu_{\Phi}^{+}(x)$. If $x \notin I$ then $\mu_{\Phi}^{+}(y+x-y) \geq p_{2}=\mu_{\Phi}^{+}(x), \mu_{\Phi}^{-}(y+x-y) \leq$ $n_{2}=\mu_{\Phi}^{-}(x)$. But, in either case, we know that $\mu_{\Phi}^{+}(y+$ $x-y) \geq \mu_{\Phi}^{+}(x), \mu_{\Phi}^{-}(y+x-y) \leq \mu_{\Phi}^{-}(x)$. Therefore $\Phi=\left(X ; \mu_{\Phi}^{+}, \mu_{\Phi}^{-}\right)$satisfies (BF3).

Let $x, y \in R$. We discuss the following two cases: If $y \in I$ Then $x y \in I$. Thus $\mu_{\Phi}^{+}(x y)=p_{1}=$ $\mu_{\Phi}^{+}(y), \mu_{\Phi}^{-}(x y)=n_{1}=\mu_{\Phi}^{+}(y)$. If $y \notin I$ then $\mu_{\Phi}^{+}(x y) \geq$ $p_{2}=\mu_{\Phi}^{+}(y), \mu_{\Phi}^{-}(x y) \leq n_{2}=\mu_{\Phi}^{-}(y)$. But, in either case, we know that $\mu_{\Phi}^{+}(x y) \geq \mu_{\Phi}^{+}(y), \mu_{\Phi}^{-}(x y) \leq \mu_{\Phi}^{-}(y)$. Therefore $\Phi=\left(X ; \mu_{\Phi}^{+}, \mu_{\Phi}^{-}\right)$satisfies (BF4)

Let $x, y, z \in R$. We discuss the following two cases: If $z \in I$ Then $(x+z) y-x y \in I$. Thus $\left.\mu_{\Phi}^{+}((x+z) y-x y)\right)=$ $p_{1}=\mu_{\Phi}^{+}(z), \mu_{\Phi}^{-}((x+z) y-x y)=n_{1}=\mu_{\Phi}^{+}(z)$. If $z \notin I$ then $\mu_{\Phi}^{+}((x+z) y-x y) \geq p_{2}=\mu_{\Phi}^{+}(z), \mu_{\Phi}^{-}((x+z) y-$ $x y) \leq n_{2}=\mu_{\Phi}^{-}(z)$. But, in either case, we know that $\mu_{\Phi}^{+}((x+z) y-x y) \geq \mu_{\Phi}^{+}(z), \mu_{\Phi}^{-}((x+z) y-x y) \leq \mu_{\Phi}^{-}(z)$. Therefore $\Phi=\left(X ; \mu_{\Phi}^{+}, \mu_{\Phi}^{-}\right)$satisfies (BF5) Therefore $\Phi=\left(X ; \mu_{\Phi}^{+}, \mu_{\Phi}^{-}\right)$is a bipolar fuzzy ideal of $R$.

Theorem 3.5. Let $\Phi=\left(R ; \mu_{\Phi}^{+}, \mu_{\Phi}^{-}\right)$be a bipolar fuzzy set in $R$. Then $\Phi=\left(R ; \mu_{\Phi}^{+}, \mu_{\Phi}^{-}\right)$is a bipolar fuzzy ideal of $R$ if and only if it satisfies the following assertions:

$$
\begin{aligned}
& (\forall t \in[0,1])\left(\Phi_{t}^{P} \neq \emptyset \Rightarrow \Phi_{t}^{P} \text { is an ideal of } R\right), \\
& (\forall s \in[-1,0])\left(\Phi_{s}^{N} \neq \emptyset \Rightarrow \Phi_{s}^{N} \text { is an ideal of } R\right) .
\end{aligned}
$$


Proof. Assume that $\Phi=\left(R ; \mu_{\Phi}^{+}, \mu_{\Phi}^{-}\right)$is a bipolar fuzzy ideal of $R$. Let $(t, s) \in[-1,0] \times[0,1]$ be such that $\Phi_{t}^{P} \neq \emptyset$ and $\Phi_{s}^{N} \neq \emptyset$. If $x, y \in \Phi_{t}^{P}$ and $a, b \in \Phi_{s}^{N}$. Then $\mu_{\Phi}^{+}(x) \geq t, \mu_{\Phi}^{+}(y) \geq t, \mu_{\Phi}^{-}(a) \leq s$ and $\mu_{\Phi}^{-}(b) \leq s$.

Now, we have

$$
\begin{aligned}
& \mu_{\Phi}^{+}(x-y) \geq \min \left\{\mu_{\Phi}^{+}(x), \mu_{\Phi}^{+}(y)\right\} \geq t, \\
& \mu_{\Phi}^{-}(a-b) \leq \max \left\{\mu_{\Phi}^{-}(a), \mu_{\Phi}^{-}(b)\right\} \leq s,
\end{aligned}
$$

and so $x-y \in \Phi_{t}^{P}$ and $a-b \in \Phi_{s}^{N}$.

Now let $x \in \Phi_{t}^{P}, a \in \Phi_{s}^{N}$ and $u, v \in R$. Then $\mu_{\Phi}^{+}(x) \geq$ $t$, and $\mu_{\Phi}^{-}(a) \leq s$ we have $\mu_{\Phi}^{+}(u+x-u) \geq \mu_{\Phi}^{+}(x) \geq t$ and $\mu_{\Phi}^{-}(v+a-v) \leq \mu_{\Phi}^{-}(a) \leq s$ which implies $u+x-u \in \Phi_{t}^{P}$ and $v+a-v \in \Phi_{s}^{N}$.

Also we have $\mu_{\Phi}^{+}(u x) \geq \mu_{\Phi}^{+}(x) \geq t$ and $\mu_{\Phi}^{-}(v a) \leq$ $\mu_{\Phi}^{-}(a) \leq s$ so that $u x \in \Phi_{t}^{\bar{P}}$ and $v a \in \Phi_{s}^{N}$.

Finally we get

$$
\mu_{\Phi}^{+}((u+x) v-u v) \geq \mu_{\Phi}^{+}(x) \geq t
$$

and

$$
\mu_{\Phi}^{-}((u+a) v-u v) \leq \mu_{\Phi}^{-}(a) \leq s
$$

which implies $(u+x) v-u v \in \Phi_{t}^{P}$ and $(u+a) v-u v \in \Phi_{t}^{N}$

Therefore $\Phi_{t}^{P}$ and $\Phi_{s}^{N}$ are ideals of $R$.

Conversely, suppose that the condition (2) is valid. If there exists $x^{\prime}, y^{\prime} \in R$ such that

$$
\mu_{\Phi}^{+}\left(x^{\prime}-y^{\prime}\right)<\min \left\{\mu_{\Phi}^{+}\left(x^{\prime}\right), \mu_{\Phi}^{+}\left(y^{\prime}\right)\right\}
$$

and

$$
\mu_{\Phi}^{-}\left(x^{\prime}-y^{\prime}\right)>\max \left\{\mu_{\Phi}^{-}\left(x^{\prime}\right), \mu_{\Phi}^{-}\left(y^{\prime}\right)\right\}
$$

then by taking

$$
\begin{aligned}
& t_{0}=\frac{1}{2}\left(\mu_{\Phi}^{+}\left(x^{\prime}-y^{\prime}\right)+\min \left\{\mu_{\Phi}^{+}\left(x^{\prime}\right), \mu_{\Phi}^{+}\left(y^{\prime}\right)\right\}\right), \\
& s_{0}=\frac{1}{2}\left(\mu_{\Phi}^{-}\left(x^{\prime}-y^{\prime}\right)+\max \left\{\mu_{\Phi}^{-}\left(x^{\prime}\right), \mu_{\Phi}^{-}\left(y^{\prime}\right)\right\}\right),
\end{aligned}
$$

we have

$$
\begin{aligned}
& \mu_{\Phi}^{+}\left(x^{\prime}-y^{\prime}\right)<t_{0}<\min \left\{\mu_{\Phi}^{+}\left(x^{\prime}\right), \mu_{\Phi}^{+}\left(y^{\prime}\right)\right\} \\
& \mu_{\Phi}^{-}\left(x^{\prime}-y^{\prime}\right)>s_{0}>\max \left\{\mu_{\Phi}^{-}\left(x^{\prime}\right), \mu_{\Phi}^{-}\left(y^{\prime}\right)\right\} .
\end{aligned}
$$

Hence $x^{\prime}-y^{\prime} \notin \Phi_{t_{0}}^{P} \cap \Phi_{s_{0}}^{N}$ and $x^{\prime}, y^{\prime} \in \Phi_{t_{0}}^{P} \cap$ $\Phi_{s_{0}}^{N}$, This is a contradiction, and so $\mu_{\Phi}^{+}(x-y) \geq$ $\min \left\{\mu_{\Phi}^{+}(x), \mu_{\Phi}^{+}(y)\right\}, \mu_{\Phi}^{-}(x-y) \leq \max \left\{\mu_{\Phi}^{-}(x), \mu_{\Phi}^{-}(y)\right\}$ for all $x, y \in R$.

If there exists $x^{\prime}, y^{\prime} \in R$ such that

$$
\mu_{\Phi}^{+}\left(x^{\prime} y^{\prime}\right)<\min \left\{\mu_{\Phi}^{+}\left(x^{\prime}\right), \mu_{\Phi}^{+}\left(y^{\prime}\right)\right\}
$$

and

$$
\mu_{\Phi}^{-}\left(x^{\prime} y^{\prime}\right)>\max \left\{\mu_{\Phi}^{-}\left(x^{\prime}\right), \mu_{\Phi}^{-}\left(y^{\prime}\right)\right\}
$$

then by taking

$$
\begin{aligned}
& t_{1}=\frac{1}{2}\left(\mu_{\Phi}^{+}\left(x^{\prime} y^{\prime}\right)+\min \left\{\mu_{\Phi}^{+}\left(x^{\prime}\right), \mu_{\Phi}^{+}\left(y^{\prime}\right)\right\}\right), \\
& s_{1}=\frac{1}{2}\left(\mu_{\Phi}^{-}\left(x^{\prime} y^{\prime}\right)+\max \left\{\mu_{\Phi}^{-}\left(x^{\prime}\right), \mu_{\Phi}^{-}\left(y^{\prime}\right)\right\}\right),
\end{aligned}
$$

we have

$$
\begin{aligned}
& \mu_{\Phi}^{+}\left(x^{\prime} y^{\prime}\right)<t_{1}<\min \left\{\mu_{\Phi}^{+}\left(x^{\prime}\right), \mu_{\Phi}^{+}\left(y^{\prime}\right)\right\} \\
& \mu_{\Phi}^{-}\left(x^{\prime} y^{\prime}\right)>s_{1}>\max \left\{\mu_{\Phi}^{-}\left(x^{\prime}\right), \mu_{\Phi}^{-}\left(y^{\prime}\right)\right\}
\end{aligned}
$$

Hence $x^{\prime} y^{\prime} \notin \Phi_{t_{1}}^{P} \cap \Phi_{s_{1}}^{N}$ and $x^{\prime}, y^{\prime} \in \Phi_{t_{1}}^{P} \cap$ $\Phi_{s_{1}}^{N}$, This is a contradiction, and so $\mu_{\Phi}^{+}(x y) \geq$ $\min \left\{\mu_{\Phi}^{+}(x), \mu_{\Phi}^{+}(y)\right\}, \mu_{\Phi}^{-}(x y) \leq \max \left\{\mu_{\Phi}^{-}(x), \mu_{\Phi}^{-}(y)\right\}$ for all $x, y \in R$.

If there exists $x^{\prime}, y^{\prime} \in R$ such that

$$
\mu_{\Phi}^{+}\left(y^{\prime}+x^{\prime}-y^{\prime}\right)<\mu_{\Phi}^{+}\left(x^{\prime}\right)
$$

and

$$
\mu_{\Phi}^{-}\left(y^{\prime}+x^{\prime}-y^{\prime}\right)>\mu_{\Phi}^{-}\left(x^{\prime}\right)
$$

then by taking

$$
\begin{aligned}
& t_{2}=\frac{1}{2}\left(\mu_{\Phi}^{+}\left(y^{\prime}+x^{\prime}-y^{\prime}\right)+\mu_{\Phi}^{+}\left(x^{\prime}\right)\right), \\
& s_{2}=\frac{1}{2}\left(\mu_{\Phi}^{-}\left(y^{\prime}+x^{\prime}-y^{\prime}\right)+\mu_{\Phi}^{-}\left(x^{\prime}\right)\right),
\end{aligned}
$$

we have

$$
\begin{aligned}
& \mu_{\Phi}^{+}\left(y^{\prime}+x^{\prime}-y^{\prime}\right)<t_{2}<\mu_{\Phi}^{+}\left(x^{\prime}\right), \\
& \mu_{\Phi}^{-}\left(y^{\prime}+x^{\prime}-y^{\prime}\right)>s_{2}>\mu_{\Phi}^{-}\left(x^{\prime}\right) .
\end{aligned}
$$

Hence $y^{\prime}+x^{\prime}-y^{\prime} \notin \Phi_{t_{2}}^{P} \cap \Phi_{s_{2}}^{N}$ and $x^{\prime} \in \Phi_{t_{2}}^{P} \cap \Phi_{s_{2}}^{N}$, This is a contradiction, and so $\mu_{\Phi}^{+}(y+x-y) \geq \mu_{\Phi}^{+}(x), \mu_{\Phi}^{-}(y+$ $x-y) \leq \mu_{\Phi}^{-}(x)$ for all $x, y \in R$.

If there exists $x^{\prime}, y^{\prime}, z^{\prime} \in R$ such that

$$
\mu_{\Phi}^{+}\left(\left(x^{\prime}+z^{\prime}\right) y^{\prime}-x^{\prime} y^{\prime}\right)<\mu_{\Phi}^{+}\left(z^{\prime}\right)
$$

and

$$
\mu_{\Phi}^{-}\left(\left(x^{\prime}+z^{\prime}\right) y^{\prime}-x^{\prime} y^{\prime}\right)>\mu_{\Phi}^{-}\left(z^{\prime}\right)
$$

then by taking

$$
\begin{aligned}
& t_{3}=\frac{1}{2}\left(\mu_{\Phi}^{+}\left(\left(x^{\prime}+z^{\prime}\right) y^{\prime}-x^{\prime} y^{\prime}\right)+\mu_{\Phi}^{+}\left(z^{\prime}\right)\right), \\
& s_{3}=\frac{1}{2}\left(\mu_{\Phi}^{-}\left(\left(x^{\prime}+z^{\prime}\right) y^{\prime}-x^{\prime} y^{\prime}\right)+\mu_{\Phi}^{-}\left(z^{\prime}\right)\right),
\end{aligned}
$$

we have

$$
\begin{aligned}
& \mu_{\Phi}^{+}\left(\left(x^{\prime}+z^{\prime}\right) y^{\prime}-x^{\prime} y^{\prime}\right)<t_{3}<\mu_{\Phi}^{+}\left(z^{\prime}\right), \\
& \mu_{\Phi}^{-}\left(\left(x^{\prime}+z^{\prime}\right) y^{\prime}-x^{\prime} y^{\prime}\right)>s_{3}>\mu_{\Phi}^{-}\left(z^{\prime}\right) .
\end{aligned}
$$

Hence $\left(x^{\prime}+z^{\prime}\right) y^{\prime}-x^{\prime} y^{\prime} \notin \Phi_{t_{3}}^{P} \cap \Phi_{s_{3}}^{N}$ and $z^{\prime} \in \Phi_{t_{3}}^{P} \cap \Phi_{s_{3}}^{N}$, This is a contradiction, and so $\left.\mu_{\Phi}^{+}((x+z) y-x y)\right) \geq$ $\left.\mu_{\Phi}^{+}(z), \mu_{\Phi}^{-}((x+z) y-x y)\right) \leq \mu_{\Phi}^{-}(z)$ for all $x, y, z \in R$.

We then $\Phi_{t}^{P}$ and $\Phi_{t}^{P}$ are called positve cut ideal and negative cut ideal of $R$. 
Corollary 3.6. If $\Phi=\left(X ; \mu_{\Phi}^{+}, \mu_{\Phi}^{-}\right)$is a bipolar-valued fuzzy ideal of $R$, then the nonempty $(t, s)$-cut $\Phi_{(s, t)}$ of $\Phi=\left(X ; \mu_{\Phi}^{+}, \mu_{\Phi}^{-}\right)$is an ideal of $R$.

We recalled that Near ring is Noetherian if it satisfied the ascending chain condition for ideal. Equivalently, every ideal is finitely generated. Equivalently, any nonempty collection of ideals has a maximal element.

Lemma 3.7. If there is an ascending chain of ideals of $R$ as following

$$
I_{0} \subset I_{1} \subset \cdots \subset I_{n}=R,
$$

then there exists an bipolar fuzzy ideals $\Phi=\left(X ; \mu_{\Phi}^{+}, \mu_{\Phi}^{-}\right)$ of $R$ whose positive cut and negative cut ideal are exactly the ideal in the above chain.

Theorem 3.8. For any near ring $R$, the following are equivalent:

(1) $R$ is Noetherian.

(2) For every bipolar fuzzy ideals $\Phi=\left(X ; \mu_{\Phi}^{+}, \mu_{\Phi}^{-}\right)$of $R$, every subset of $\operatorname{Im}\left(\mu_{\Phi}^{+}\right)$has the least element.

(3) For every bipolar fuzzy ideals $\Phi=\left(X ; \mu_{\Phi}^{+}, \mu_{\Phi}^{-}\right)$of $R$, every subset of $\operatorname{Im}\left(\mu_{\Phi}^{-}\right)$has the greatest element.

Proof. (1) $(\Rightarrow)(2)$ and (3): Suppose that there is an bipolar fuzzy ideal $\Phi=\left(X ; \mu_{\Phi}^{+}, \mu_{\Phi}^{-}\right)$and a subset of $\operatorname{Im}\left(\mu_{\Phi}^{+}\right)$ has not the least element. Then there exists a strictly decreasing sequence $\left\{t_{n}\right\}$ which has no the least element and a sequence $\left\{x_{n}\right\}$ in $R$ such that $\mu_{\Phi}^{+}\left(x_{n}\right)=t_{n}$. Let $B_{n}:=\left\{x \in R: \mu_{\Phi}^{+}(x) \geq t_{n}\right\}$. Then $B_{1} \subsetneq B_{2} \subsetneq \cdots$ is a strictly ascending chain of dieals of $R$, contradicting the assumption that $R$ is Noetherian. Therefore every subset of $\operatorname{Im}\left(\mu_{\Phi}^{+}\right)$has the least element for any bipolar fuzzy ideal $\Phi=\left(X ; \mu_{\Phi}^{+}, \mu_{\Phi}^{-}\right)$of $R$. By similar method, we get that every subset of $\operatorname{Im}\left(\mu_{\Phi}^{-}\right)$has the greatest element for any bipolar fuzzy ideal $\Phi=\left(X ; \mu_{\Phi}^{+}, \mu_{\Phi}^{-}\right)$of $R$. (2) or (3) ( $\left.\Rightarrow\right)$ (1): Suppose that $R$ is not Noetherian. Then there exists a strictly ascending chain $G_{0} \subset G_{1} \subset G_{2} \subset \cdots$ of ideals of $R$ which does not terminate at finite step. Define a biplar fuzzy set $\Phi=\left(X ; \mu_{\Phi}^{+}, \mu_{\Phi}^{-}\right)$in $R$ by

$$
\begin{gathered}
\mu_{\Phi}^{+}(x):= \begin{cases}\frac{1}{k+1} & \text { if } x \in G_{k} \backslash G_{k-1}, \\
0 & \text { if } x \notin \bigcup_{k=1}^{\infty} G_{k},\end{cases} \\
\mu_{\Phi}^{-}(x):= \begin{cases}-\frac{1}{k+1} & \text { if } x \in G_{k} \backslash G_{k-1}, \\
0 & \text { if } x \notin \bigcup_{k=1}^{\infty} G_{k},\end{cases}
\end{gathered}
$$

We prove that $\Phi=\left(X ; \mu_{\Phi}^{+}, \mu_{\Phi}^{-}\right)$is an bipolar fuzzy ideal of $R$. Let $x, y \in R$. If $x, y \in \bigcup_{k=1}^{\infty} G_{k}$. In this case, we assume that $x \in G_{l} \backslash G_{l-1}$ and $y \in G_{m} \backslash$ $G_{m-1}$ for $l=0,1,2, \cdots ; m=1,2, \cdots$. Without loss of generality, we may assume that $l \leq m$. Then obviously $x \in G_{m}$, and so $x-y, x y \in G_{m}$ because
$G_{m}$ is a bipolar ideal of $R$. It follows that $\mu_{\Phi}^{+}(x-$ $y) \geq \frac{1}{m+1}=\min \left\{\frac{1}{m+1}, \frac{1}{l+1}\right\}=\min \left\{\mu_{\Phi}^{+}(x), \mu_{\Phi}^{+}(y)\right\}$ and $\mu_{\Phi}^{-}(x-y) \leq-\frac{1}{m+1}=\max \left\{-\frac{1}{m+1},-\frac{1}{l+1}\right\}=$ $\max \left\{\mu_{\Phi}^{-}(x), \mu_{\Phi}^{-}(y)\right\}$, Also we have $\mu_{\Phi}^{+}(x y) \geq \frac{1}{m+1}=$ $\min \left\{\frac{1}{m+1}, \frac{1}{l+1}\right\}=\min \left\{\mu_{\Phi}^{+}(x), \mu_{\Phi}^{+}(y)\right\}$ and $\mu_{\Phi}^{-}(x y) \leq$ $-\frac{1}{m+1}=\max \left\{-\frac{1}{m+1},-\frac{1}{l+1}\right\}=\max \left\{\mu_{\Phi}^{-}(x), \mu_{\Phi}^{-}(y)\right\}$,

If $x$ or $y$ is not contained in $\bigcup_{k=1}^{\infty} G_{k}$, then clearly $\min \left\{\mu_{\Phi}^{+}(x), \mu_{\Phi}^{+}(y)\right\}=0$ and $\max \left\{\mu_{\Phi}^{-}(x), \mu_{\Phi}^{-}(y)\right\}=0$. Then $\mu_{\Phi}^{+}(x-y) \geq \min \left\{\mu_{\Phi}^{+}(x), \mu_{\Phi}^{+}(y)\right\}, \mu_{\Phi}^{-}(x-y) \leq$ $\max \left\{\mu_{\Phi}^{-}(x), \mu_{\Phi}^{-}(y)\right\}, \mu_{\Phi}^{+}(x y) \geq \min \left\{\mu_{\Phi}^{+}(x), \mu_{\Phi}^{+}(y)\right\}$ and $\mu_{\Phi}^{-}(x y) \leq \max \left\{\mu_{\Phi}^{-}(x), \mu_{\Phi}^{-}(y)\right\}$. Therefore $\Phi=$ $\left(X ; \mu_{\Phi}^{+}, \mu_{\Phi}^{-}\right)$satisfies (BF1) and (BF2).

Let $x, y \in R$. We discuss the following two cases: If $x \in \bigcup_{k=1}^{\infty} G_{k}$, then there exists $k \in \mathbb{N}$ such that $x \in$ $G_{k} \backslash G_{k-1}$. Then $y+x-y \in G_{k}$ Thus $\mu_{\Phi}^{+}(y+x-y) \geq$ $\frac{1}{k+1}=\mu_{\Phi}^{+}(x), \mu_{\Phi}^{-}(y+x-y) \leq-\frac{1}{k+1}=\mu_{\Phi}^{-}(x)$. If $x \notin \bigcup_{k=1}^{\infty} G_{k}$, then $\mu_{\Phi}^{+}(y+x-y) \geq 0=\mu_{\Phi}^{+}(x), \mu_{\Phi}^{-}(y+$ $x-y) \leq 0=\mu_{\Phi}^{-}(x)$. But, in either case, we know that $\mu_{\Phi}^{+}(y+x-y) \geq \mu_{\Phi}^{+}(x), \mu_{\Phi}^{-}(y+x-y) \leq \mu_{\Phi}^{-}(x)$. Therefore $\Phi=\left(X ; \mu_{\Phi}^{+}, \mu_{\Phi}^{-}\right)$satisfies (BF3).

Let $x, y \in R$. We discuss the following two cases: If $y \in$ $\bigcup_{k=1}^{\infty} G_{k}$, then there exists $k \in \mathbb{N}$ such that $y \in G_{k} \backslash G_{k-1}$. Then $x y \in G_{k}$ Thus $\mu_{\Phi}^{+}(x y) \geq \frac{1}{k+1}=\mu_{\Phi}^{+}(y), \mu_{\Phi}^{-}(x y) \leq$ $-\frac{1}{k+1}=\mu_{\Phi}^{-}(y)$. If $y \notin \bigcup_{k=1}^{\infty} G_{k}$, then $\mu_{\Phi}^{+}(x y) \geq 0=$ $\mu_{\Phi}^{+}(y), \mu_{\Phi}^{-}(x y) \leq 0=\mu_{\Phi}^{-}(y)$. But, in either case, we know that $\mu_{\Phi}^{+}(x y) \geq \mu_{\Phi}^{+}(y), \mu_{\Phi}^{-}(x y) \leq \mu_{\Phi}^{-}(y)$. Therefore $\Phi=\left(X ; \mu_{\Phi}^{+}, \mu_{\Phi}^{-}\right)$satisfies (BF4).

Let $x, y, z \in R$. We discuss the following two cases: If $z \in \bigcup_{k=1}^{\infty} G_{k}$, then there exists $k \in \mathbb{N}$ such that $z \in G_{k} \backslash G_{k-1}$. Then $x y \in G_{k}$ Thus $\mu_{\Phi}^{+}((x+z) y-x y) \geq$ $\frac{1}{k+1}=\mu_{\Phi}^{+}(z), \mu_{\Phi}^{-}((x+z) y-x y) \leq-\frac{1}{k+1}=\mu_{\Phi}^{-}(z)$. If $z \notin \bigcup_{k=1}^{\infty} G_{k}$, then $\mu_{\Phi}^{+}((x+z) y-x y) \geq 0=$ $\mu_{\Phi}^{+}(z), \mu_{\Phi}^{-}((x+z) y-x y) \leq 0=\mu_{\Phi}^{-}(z)$. But, in either case, we know that $\mu_{\Phi}^{+}((x+z) y-x y) \geq \mu_{\Phi}^{+}(z), \mu_{\Phi}^{-}((x+$ $z) y-x y) \leq \mu_{\Phi}^{-}(z)$. Therefore $\Phi=\left(X ; \mu_{\Phi}^{+}, \mu_{\Phi}^{-}\right)$satisfies (BF5).

Therefore $\Phi=\left(X ; \mu_{\Phi}^{+}, \mu_{\Phi}^{-}\right)$is a bipolar fuzzy ideal of $R$. Since $\operatorname{Im}\left(\mu_{\Phi}^{+}\right)=\left\{\frac{1}{k+1} \mid k=0,1,2, \cdots\right\}$ and $\operatorname{Im}\left(\mu_{\Phi}^{-}\right)=\left\{-\frac{1}{k+1} \mid k=0,1,2, \cdots\right\}$, any subset of $\operatorname{Im}\left(\mu_{\Phi}^{+}\right)$has no the least element and any subset of $\operatorname{Im}\left(\mu_{\Phi}^{-}\right)$has no the greatest elements. This is contradiction for either case. Therefore $R$ is Noetherian.

\section{Conclusion}

In this paper we studied the algebraic properties of bipolar fuzzy set in Near ring structures. This work focused on bipolar fuzzy subring/ideal of a Near ring. Finally, we considered the structure of Noetherian Near ring by using these bipolar fuzzy ideals. In our future study, we will consider the bipolar fuzzy prime(semiprime) ideals and quotient of Near ring by using these bipolar fuzzy ideals will be pre- 
sented.

\section{References}

[1] Interval-Valued H-Fuzzy Sets, Lee, Keon-Chang; Lee, Jeong-Gon; Hur, Kul / International Journal of Fuzzy Logic and Intelligent Systems, v.10, no.2, pp.134-141, 2010.

[2] S. Abou-Zaid, On fuzzy subnear-rings and ideals, Fuzzy Sets and Sys., Vol. 44,No.1,pp.139-146,1991.

[3] M.Akram Bipolar fuzzy graphs Information Sciences, Vol. 181, No.24, pp. 5548-5564, 2011.

[4] Y. B. Jun and S. Z. Song, Subalgebras and closed ideals of BCH-algebras based on bipolar-valued fuzzy sets, Sci. Math. Jpn., Vol.68, No.2, pp.287-297, 2008.

[5] K.C. Lee,J.G Lee, K.Hur, Interval-Valued H-Fuzzy Sets, International Journal of Fuzzy Logic and Intelligent Systems, Vol.10, No.2, pp.134-141, 2010.

[6] K. J. Lee, Bipolar fuzzy subalgerbas and bipolar fuzzy ideals of BCK/BCI-algerbas,Bull. Malays. Math. Sci. Soc., Vol.32, No.3, pp.361-373,2009.

[7] Y. B. Jun and C. H. Park, Filters of BCH-Algebras based on bi-polar-valued fuzzy sets, Int. Math. Forum, Vol.14.,No.13,pp.631-643, 2009.

[8] Y. B. Jun and J. Kavikumar, Bipolar fuzzy finite state machines,Bull. Malays. Math. Sci.Soc. Vol,34,No.1, pp.181-188,2011.

[9] K. M. Lee, Bipolar-valued fuzzy sets and their operations, Proc. Int. Conf. on Intelligent Technologies, Bangkok, Thailand, pp.307-312, 2000.
[10] K. M. Lee, Comparison of interval-valued fuzzy sets, intuitionistic fuzzy sets, and bipolar-valued fuzzy sets, J. Fuzzy Logic Intelligent Systems, Vol.14,No.2, pp.125-129, 2004.

[11] J.H.Park, Y.C.Kwun, M.J.Son, A Generalized Intuitionistic Fuzzy Soft Set Theoretic Approach to Decision Making Problems International Journal of Fuzzy Logic and Intelligent Systems, Vol.11, No.2, pp.7176, 2011.

[12] J.H.park,Operations on Generalized Intuitionistic Fuzzy Soft Sets International Journal of Fuzzy Logic and Intelligent Systems, Vol.11, No.3, pp.184-189, 2011.

[13] G.Pilz, Near-Rings, North-Holland Publishing Company,Amsterdam ,New York-Oxford,1983.

[14] L. A. Zadeh,Fuzzy sets, Inform. and Control, Vol.8. No.3, pp.338-353, 1965.

\section{저 자 소 개}

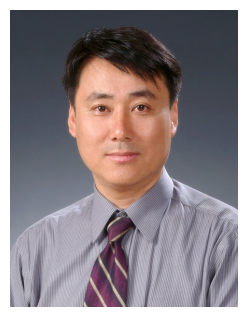

Hyoung Gu Baik Professor of the School of Computer and Information, Ulsan Colleage Research Area: Operator Theory, Fuzzy Theory E-mail : hgbaik@uc.ac.kr 